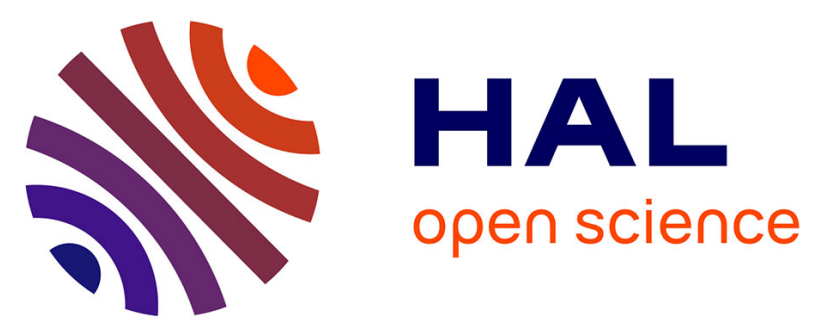

\title{
Europium labeled lactosylated albumin as a model workflow for the development of biotherapeutics
}

Hugo Salmon, Rabah Gahoual, Pascal Houzé, Tayssir Ibrahim, Michel Bessodes, Daniel Scherman, Johanne Seguin, Nathalie Mignet

\section{- To cite this version:}

Hugo Salmon, Rabah Gahoual, Pascal Houzé, Tayssir Ibrahim, Michel Bessodes, et al.. Europium labeled lactosylated albumin as a model workflow for the development of biotherapeutics. Nanomedicine: Nanotechnology, Biology and Medicine, 2019, 18, pp.21-30. 10.1016/j.nano.2019.02.011 . hal02123126

\section{HAL Id: hal-02123126 \\ https://hal.science/hal-02123126}

Submitted on 25 Oct 2021

HAL is a multi-disciplinary open access archive for the deposit and dissemination of scientific research documents, whether they are published or not. The documents may come from teaching and research institutions in France or abroad, or from public or private research centers.
L'archive ouverte pluridisciplinaire HAL, est destinée au dépôt et à la diffusion de documents scientifiques de niveau recherche, publiés ou non, émanant des établissements d'enseignement et de recherche français ou étrangers, des laboratoires publics ou privés.

\section{(ㅇ)(1) $\$$}

Distributed under a Creative Commons Attribution - NonCommerciall 4.0 International 


\section{Article type: research article}

Word counts

- abstract: 150

- complete manuscript: 4874

- number of references: 28

- number of figures: 6

- number of tables: 1

Europium labelled lactosylated albumin as a model workflow for the development of biotherapeutics H. Salmon ${ }^{\mathrm{a}, 1}$, R. Gahoual ${ }^{\mathrm{a}}$, P. Houzé, ${ }^{\mathrm{a}, \mathrm{b}}$, T. Ibrahim ${ }^{\mathrm{a}}$, M. Bessodes ${ }^{\mathrm{a}}$, D. Scherman $^{\mathrm{a}}$, J. Seguin ${ }^{\mathrm{a}, \mathrm{c}}$, N. Mignet ${ }^{\mathrm{a} *}$

${ }^{a}$ Chemical and Biological Technologies for Health (UTCBS), CNRS UMR8258, INSERM U1022, Paris Descartes University, Sorbonne-Paris-Cité, Chimie ParisTech, PSL Research University, School of Pharmacy, 4 avenue de l'observatoire, F-75006 Paris, France E-mail: nathalie.mignet@parisdescartes.fr

${ }^{b}$ Laboratoire de Biochimie, Hôpital Universitaire Necker-Enfants malades, Assistance Publique-Hôpitaux de Paris (AP-HP), Paris, France

Dr. J.S. Author 7

${ }^{c}$ Optical imaging platform LIOPA, Life imaging, PIV, Faculty of Pharmacy, Paris Descartes University, Paris, France

${ }^{1}$ Present address: McGill University, Genoma Building, Dept. of Biomedical Engineering, 740 Dr. Penfield Drive, Room 4300, Montréal, QC, CANADA.

Abbreviations: Human serum albumin (HSA); Lactosylated-DTPA albumin (LACTAL): asymmetrical flow field-flow fractionation (AF4); size exclusion chromatography (SEC); refractive index (RI); asialoglycoprotein (ASGP); ASGP receptor (ASGPR), molar weight (MW), light scattering (LS).

Keywords: targeted imaging; nanomedicines; bioconjugates development; lanthanide chelates; time resolved fluorescence

Abstract. Lactosylated albumin is currently used as a radiopharmaceutical agent to image the liver asialoglycoprotein receptors and quantify hepatic liver function in various diseases. A lactosylated protein (LACTAL) conjugate showed excellent liver uptake compared to nonlactosylated protein and a high signal to noise ratio, based on the biodistribution in mice using ${ }^{99 m}$ Tc-scintigraphy. However, in the laboratory, it is useful to have a method that can be used in daily practice to quantify cellular targeting or biodistribution. We propose a methodology from synthesis validation to pre-clinical demonstration and introduce a new practical detector (LACTAL.Eu) of the LACTAL molecule in biological media. We confirmed the purity and colloidal stability of the sample through physical analytical techniques, then showed the absence of in vitro toxicity of the agent and demonstrated in vitro targeting. Taking advantage 
of the fluorescence decay of the lanthanide, we performed measurements directly on the cell media without any further treatment. Finally, biodistribution in mice was confirmed by ex vivo measurements.

\section{Background}

The use of proteins for therapeutic purpose has proven to be an unprecedented success over the last two decades and has spurred the growth of the biopharmaceutical industry. The number of proteins approved and technologies involved continue to increase. ${ }^{1}$ One of the most advanced technologies is the chemical modification of proteins to incorporate additional functionalities. In particular, the use of chemical modification has successfully produced antibody-drug conjugates that combine the epitope specificity of immunoglobulin $\mathrm{G}$ (IgG) with a potent cytotoxic drug covalently bonded to the peptide backbone. ${ }^{2}$

Currently, no rigorous method exists to relate the chemical identity of a protein to its biological one, especially by evaluating its in vivo outcome. ${ }^{3}$ Nanotechnology Characterization Laboratories (NCL) have been created both in the US and Europe for the development of standardized analytical methods. ${ }^{4,5}$ As a result of the inherent complexity of proteins, partial modifications to the peptide backbone require detailed characterization after the modification process. Indeed, the addition of a chemical compound, covalently bonded to the peptide backbone, could potentially destabilize the tertiary and/or quaternary structure of the native protein. Therefore, it could potentially result in a drastic modification of the physico-chemical properties and functionality of the protein. For instance, protein aggregation has been found to be primarily due to partial unfolding. ${ }^{6}$ Consequently, intensive research was recently conducted to establish the analytical methodologies required to achieve a comprehensive characterization of the different levels defining the structure of therapeutic proteins. ${ }^{7,8}$ These properties should provide a global understanding of the structure of the developed therapeutic protein to assess the formation of endogenous species such as 
aggregates and/or other types of degradation products. ${ }^{9}$ Thus, from a development perspective, the implementation of a dedicated analytical workflow should also provide robust information regarding potential protein conjugates to optimize the modification process. Such a methodology could be useful to nanomaterial development ranging from ongoing fundamental research to preclinical evaluations.

Prior to the clinical phase, the biodistribution of therapeutic proteins should be assessed, especially when the protein strategy involves specific targeting. In general, biodistribution is performed by positron emission tomography (PET) or single-photon emission tomography (SPET) imaging due to their high sensitivity, or even single-photon emission computed tomography (SPECT) imaging where both resolution and sensitivity is obtained. However, labelling conditions are a main concern since they can alter protein structure or induce aggregation. ${ }^{10}$ Moreover, the use of radioisotopes requires additional safety and experimental conditions that are not available in all laboratories. In this context, we investigated the shift to lanthanide labelling as a means of anticipating labelling conditions and protein characterization. As such, Europium $(\mathrm{Eu})$ appears to be a good candidate for substitution. Eu (III) is readily available, does not require a restricted lifetime compared to conventional fluorophores, and has also demonstrated strong photoluminescent capacity. ${ }^{12}$ Eu (III) forms complexes with metal cation chelating agents like diethylenetriamine-pentaacetic acid (DTPA) or tetraazacyclododecane-tetraacetic acid (DOTA), so it could be used to replace ${ }^{99 \mathrm{~m}} \mathrm{Tc}$ or ${ }^{68} \mathrm{Ga}$ to obtain preliminary in vitro and in vivo data.

For liver disease patients, the assessment of hepatic function plays a decisive role in developing the appropriate therapeutic strategy, which might include irreversible measures like partial resection and liver transplantation. ${ }^{13}$ Patients with primary and secondary liver diseases demonstrate a reduced number of asialoglycoprotein (ASGP) receptors, expressed mainly on the hepatocyte sinusoidal surface. ${ }^{14}$ ASGP receptors allow for the internalization of ASGP through receptor mediated endocytosis, so they are a relevant indicator of functional 
hepatocytes. The quantification of liver uptake using radiolabeled ASGP, in the format of ${ }^{99 \mathrm{~m}}$ Tc-DTPA-galactosyl serum albumin $\left({ }^{99 \mathrm{~m}} \mathrm{Tc}-\mathrm{GSA}\right)$, proved to be a reliable and noninvasive approach for the evaluation of liver function. ${ }^{15}$ We have previously described a novel one-pot preparation protocol of ${ }^{99 \mathrm{~m}} \mathrm{Tc}-\mathrm{DTPA}-$ lactosyl serum albumin (LACTAL). ${ }^{16}$ For LACTAL preparation, two different chemical compounds were conjugated to the protein. DTPA was incorporated onto the HSA peptide backbone to form complexes with the ${ }^{99 \mathrm{~m}} \mathrm{Tc}$. Targeting of the ASGP receptor was provided by the grafting of lactose moieties. The labelling process maintained the stability of human serum albumin (HSA) to provide consistent liver targeting. Radiolabeled HSA showed interesting liver targeting capabilities. In addition, the detected signal in scintigraphy provided semi-quantitative information regarding the uptake kinetics. ${ }^{17}$

In this work, we first implemented $\mathrm{Eu}$ (III) for the substitution of ${ }^{99 \mathrm{~m}} \mathrm{Tc}$ to the LACTAL protein conjugate, providing a daily labor practice tool. We performed detailed characterization using orthogonal analytical techniques. We estimated the mean number of each type of chemical compound (propyl-DTPA and propyl-lactose) effectively added to HSA. The formation of endogenous aggregates due to the modification of the protein was evaluated. LACTAL was successfully used for liver scintigraphy imaging, so it demonstrates the relevance of $\mathrm{Eu}$ (III) use as a fluorescent label. A panel of in vitro experiments were conducted to demonstrate that Eu (III) can successfully form complexes with the chelating agent grafted to the protein and do not provide cytotoxicity. Concomitantly, in vivo biodistribution was tracked to demonstrate the applicability of Eu (III) fluorescence in biological media. These experiments also allowed for the determination of whether specific hepatocyte targeting could be maintained when $\mathrm{Eu}$ (III) replaced the ${ }^{99 \mathrm{~m}} \mathrm{Tc}$ moiety in the probe. We finally introduced an original workflow spanning the synthesis and development of LACTAL.Eu to in-vivo validation, and offer it as a guideline to develop biomolecular conjugates for imaging applications, specifically using lanthanide cryptates. Each step 
included go/no-go tests and standard methods to illustrate the workflow. We emphasized analytical validation to quantify grafting and colloidal stability.

\section{Methods}

Chemicals reagents were obtained from Sigma-Aldrich ((Breda, The Netherlands), solvents were purchased from Carlo Erba (Val-de-Reuil, France). Maleimido-propyl (1-( $\beta$-DLActosyl)-3-maleimidopropionamide) lactose was synthetized as described earlier. ${ }^{17}$ Maleimide-DTPA (2,2'-(1-carboxy-2-(carboxymethyl)-13-(2,5-dioxo-2,5-dihydro-1H-pyrrol1-yl)-10-oxo-2,5,8,11-tetraazatridecane-5,8-diyl) diacetic acid) was purchased from Chematech (France). Human serum albumin compliant with European safety regulation was purchased as Ydralbum from LFB (France) as a $200 \mathrm{mg} / \mathrm{mL}$ solution in normal saline. Disposable $0.22 \mu \mathrm{m}$ Stericup filter units were purchased from Merck-Millipore (Molsheim, France). Ultra-centrifugal concentrators Vivaspin 5, 30 kDa MWCO and Vivaspin 500, $30 \mathrm{kDa}$ MWCO were from Sartorius. Dilutions in water were achieved using milliQ water, $18.2 \mathrm{M} \Omega . \mathrm{cm}-1$.

\section{Asymmetrical flow field flow fractionation coupled to multi-angle light scattering} analysis, UV and RI (AF4-MALS-UV-RI) characterization. The AF4 controlling system was an Eclipse Dual Tec separation module from Wyatt Technology Europe, Germany, suitable for aqueous systems. The mobile phase was delivered using a Thermo Fischer scientific Dionex Ultimate 3000 HPLC (Manchester, UK) natively equipped with a vacuum degasser, an auto-sampler and a UV detector. Between the auto-sampler and HPLC pump, mobile phase was filtered in-line by a $0.1 \mu \mathrm{m}$ durapore PVDF membrane in a PEEK in-line filter. This channel was an SC series (15 cm long) with a wide type spacer of $350 \mu \mathrm{m}$. The membranes used for separation were made of regenerated cellulose, Wyatt Technology. Detection was realized using UV at a wavelength of $280 \mathrm{~nm}$. Concomitantly, refractive index quantifications were performed by means of a T-Rex differential refractometer detector using a $660 \mathrm{~nm}$ laser source (Wyatt Technology). Mobile phase was NaNO3 $10 \mathrm{mM} \mathrm{NaN3} 0.02 \%$ 
in milli-Q water degassed by vacuum pump and filtered using stericup filter units. Bioconjugates were eluted with a two-step separation. First step - focalization - was an isoforce (constant flow rate) of $3 \mathrm{~mL} / \mathrm{min}$ during $5 \mathrm{~min}$ and second was an exponential decrease of the separative force from $3 \mathrm{~mL} / \mathrm{min}$ to $0 \mathrm{~mL} / \mathrm{min}$ in $9 \mathrm{~min}$. The preliminary $5 \mathrm{~min}$ focusing step at $3 \mathrm{~mL} / \mathrm{min}$ limits diffusion effect to maximize the separation resolution. The aggregation comparative study used standard clinical HSA as a control and all solutions were injected at $2 \mathrm{mg} / \mathrm{mL}$ in $50 \mu \mathrm{L}$. MALS detection was achieved with a DAWN module from Wyatt Technology Europe. It was equipped with a Qels module for DLS supplementary analysis at angle 9 of the MALS detector. Data analysis was achieved on Astra 6.1, Wyatt Technology.

Mass Spectrometry (MS) characterization. MS experiments were performed using a Voyager DE pro mass spectrometer from Applied Biosystems (Darmstadt, Germany). This instrument implements a matrix assisted laser desorption (MALDI) ionization source and a time-of-flight (TOF) mass analyzer. The matrix used was composed of $\alpha$-Cyano-4hydroxycinnamic acid (HCCA) purchased from Sigma-Aldrich (Breda, The Netherlands). Based on bicinchoninic acid assay results, the samples were diluted to a final concentration of $1 \mu \mathrm{g} / \mu \mathrm{L}$ in milli-Q water. A sample volume of $1 \mu \mathrm{L}$ was mixed with $3 \mu \mathrm{L}$ of HCCA ( $10 \mathrm{mg} / \mathrm{mL}$ in $\mathrm{H}_{2} \mathrm{O} / \mathrm{ACN}$ ), then $1 \mu \mathrm{L}$ of this mixture was deposited on a stainless-steel plate. The droplet was left to dry at room temperature. MS measurements were performed in positive ionization and linear mode. Ion transfer parameters were optimized using the clinical grade human serum albumin. MS spectra were acquired in the $\mathrm{m} / \mathrm{z}$ range $8400-101000 \mathrm{Th}$. Each MS spectrum was obtained through the summation of 500 laser shots. MS data treatment was performed using Voyager Data explorer software (Applied Biosystems, Foster city, CA). The average mass, for the detected species was determined by considering the different charge states detected, usually from 1 to 3. 
Ricin agglutinin test: Different concentration of Lactal.Eu or HSA-DTPA.Eu and ricin agglutinin $(1 \mathrm{~g} / \mathrm{l})$ were prepared by mixing equal volumes. The turbidity was then measured at $450 \mathrm{~nm}$ with a Victor2 spectrophotometer plate reader from Perkin-Elmer.

HEPG2 internalization. HEPG2 cells were grown into DMEM supplemented with 1glutamin $(29.2 \mathrm{mg} / \mathrm{mL})$, penicillin $(50 \mathrm{U} / \mathrm{mL})$, streptomycin $(50 \mathrm{U} / \mathrm{mL})$, and $10 \%$ fetal bovine serum. The day before the experiment, the cells were seeded into 24 well culture plates at 3 different concentrations $\left(10^{5}, 2 \times 10^{5}, 4 \times 10^{5}\right)$ overnight at $37^{\circ} \mathrm{C}$ under $5 \% \mathrm{CO}$. Twenty-four hours later HSA-DTPA.Eu and LACTAL.Eu (6.2 $\mu \mathrm{M}$ DTPA) were added for four hours at $37^{\circ} \mathrm{C}$. Europium signals were measured in the cells lysate with a spectrofluorometer (Victor, Perkin Elmer). The amount of DTPA in LACTAL.Eu or DTPA.Eu in the cell lysate was calculated to the respective value obtained from the cells taken as $100 \%$ with calibration curves realized in the same condition.

In vivo study. Experiments were conducted following the Guidelines for Care and Use of Laboratory Animals of European directive No. 2010-63 and French decree No. 2013-118 and in agreement with a regional ethics committee for animal experimentation (Animal Ethics Committee of Paris Descartes No. 34 , CEEA 34) under the project number APAFiS \# 13636. Six-week-old female ( $\approx 20 \mathrm{~g}$ ) BALB/c J mice (Janvier) were inserted in a rodent restrainer and HSA-DTPA.Eu or LACTAL.EU were injected into the mouse tail vein $(200 \mu 1,2.7 \mathrm{mg} / \mathrm{ml}$ for LACTAL.Eu and $2 \mathrm{mg} / \mathrm{mL}$ for HSA-DTPA.Eu). One mouse was dedicated as a control, 5 were injected per product. Twenty minutes after injection we proceeded to euthanasia, perfused PBS into the heart left ventricle and gathered the first drops of concentrated blood by cutting the right atrium. PBS injection was achieved using a peristaltic pump at $5 \mathrm{~mL} / \mathrm{min}$. After $25-30 \mathrm{~mL}$ perfused in mice to remove blood, the organs appeared bleached. We then collected and weighed the liver, spleen, lungs and kidney.

Probe extraction. After collecting the tissues, we immersed organs in Luciferase Cell Culture Lysis 5x Reagent (125 mM Tris (pH 7.8) with H3PO4, $10 \mathrm{mM}$ CDTA, $10 \mathrm{mM}$ DTT, $50 \%$ 
glycerol and $5 \%$ Triton X-100) mixed with a Red Blood Cell Lysis Solution 1X Miltenyi Biotec. We took into consideration each organ's measured relative density (liver 0.99, spleen 1.01, kidney 0.97, lung 0.60). We performed tissue homogenizing using a Precellys Evolution manufactured by Bertin Instruments and supplied by Ozyme (Montigny-Le-Bretonneux, France). Lysis kits were 2 and $7 \mathrm{~mL}$ tubes, containing $1.4 \mathrm{~mm}$ ceramic beads (CK14). 4 cycles of $30 \mathrm{~s}$ were achieved at $6500 \mathrm{rpm}$ with samples being maintained $1 \mathrm{~min}$ on ice between each cycle to avoid bubble formation and heat degradation.

\section{Results}

Synthesis and analytical assessment of LACTAL.Eu. LACTAL was verified based on amine thiolation using Traut's reagent followed by thiol maleimide reaction (Figure 1) and was improved compared to previous $\operatorname{art}^{17}$ in favor of lactose binding over DTPA. First by optimizing the thiolation of lysine hot spots (Figure 1A), then by introducing the lactose with a 15 min delay before DTPA, - Figure 1B. Remaining free maleimide-DTPA may chelate free $\mathrm{Eu}$ (III), therefore it had to be removed from the sample before the addition of Eu (III) using ultrafiltration. To evaluate the eventual presence of free DTPA, Eu (III) was added to the filtrates and the solution was measured by time-resolved fluorimetry (TRF) to ensure that all free DTPA had been washed out (Figure S1). 4 filtration cycles led to a more than $99.9 \%$ drop in the fluorescent signal. The final medium of the chelates was saline buffer to avoid competitive complexation with ions such as phosphate during conservation which could lead to $\mathrm{Eu}(\mathrm{III})$ transfer. ${ }^{18}$

We then evaluated the global synthesis yield and aggregation distribution. The average efficiency of synthesis could be first evaluated by a bicinchonic acid assay (BCA) - Figure S2. Below $\mathrm{R}<80 \%$, the reaction efficiency was considered unsatisfying and led to the optimization of reactive quantities, ultracentrifugation time and speed and membrane material used, mainly to avoid precipitates. LACTAL synthesis with optimized conditions reached upto $90 \%$. 
Figure 2B summarizes the distribution of sizes up-to trimer sizes and aggregates (usually above $10 \mathrm{~nm}$ aggregated objects) and confirms that $80 \%$ of LACTAL as well as HSA-DTPA remained under its monomeric form. This hyphenated analysis could also be a substitute to protein quantification by UV or RI detection by achieving preliminary calibrations. This technique also revealed that DTPA grafting seemed to generate more aggregates as LACTAL and HSA-DTPA observe a 10-14\% fraction whereas lactosylated and non-modified albumin displayed better stability. This is most likely due to DTPA being highly sensitive to $\mathrm{pH}$ variations. An optimization of buffer $\mathrm{pH}$ and salinity could improve performances.

The ligand quantification average value could be achieved using commercial assay kits as described previously: thiol can be evaluated using the Ellman's reagent and maleimide using a reverse glutathione assay. ${ }^{17}$ The accuracy and specificity of these assays vary greatly depending on reagents. While an average ligand concentration is provided, there is no information about the monomer polydispersity induced by grafting. We hereby proposed to base our study on physical analysis methods and separation techniques.

We used hyphenated method AF4 (or SEC)-MALS-UV-RI performed using light scattering to obtain aforementioned aggregate quantification and molar weight increase of each synthesis product in a single run. From the Zimm scattering plot, we extracted the molar mass increase and compared it to MS values - see Figure 2C. We plotted in Figure 2A the MW function of elution volume and observed a slight variation on the monomer peak due to inhomogeneity generated by synthesis mechanism. This homogeneity was even more evident when we studied aggregates, whose MW varied between $200 \mathrm{kDa}$ and $1 \mathrm{MDa}$. Gyration radii of samples could also be monitored from Zimm plot above 10nm in Figure 2D. This allowed for the control of aggregate sizes and confirmation that they remained between 10 and $100 \mathrm{~nm}$. Averaged MW values for each HSA monomer (HSA, HSA-Lactose, LACTAL and HSADTPA) were summed up in Table 1 (LS column). 
The average number of each compound, effectively coupled to HSA, was also determined using MALDI-TOF mass spectrometry which allowed for the estimation of induced polydispersity from a few $\mu \mathrm{g}$ of sample as well as the MW increase to assess the grafted mass. Thus, as illustrated by Figure 3A, the MS spectrum corresponding to the clinical HSA gave an average mass of $66447 \mathrm{Da}$ which agrees with the theoretical mass of HSA considering the performances of the instrument. The characterization of the HSA-DTPA conjugate allowed measurement of a mean mass of $76210 \mathrm{Da}$ which represents an additional mass of $9763 \mathrm{Da}$ compared to the native HSA (Figure 3B). Using this mass difference, it was possible to estimate the attribution of a mean grafting value of 12 DTPA per protein. Also, the results indicated that 15 additional lysines underwent thiolation but did not react with the maleimideDTPA. The experiment performed on the LACTAL.Eu conjugate allowed for measurement of an average mass of $76610 \mathrm{Da}$ as emphasized in Figure 3C. The mass difference compared to native HSA was attributed to the incorporation of 7 DTPA and 7 lactoses onto the peptide backbone of the protein. Also, like the previous conjugates, 12 additional lysines were found to be modified through thiolation but did not ultimately undergo incorporation of either one of the ligands. The results achieved using MALDI-TOF mass spectrometry demonstrated that it is possible to obtain relevant and consistent information in a straightforward manner regarding the conjugation process, which enables critical evaluation of the performance of the conjugation reaction.

MALDI-TOF MS characterization could be therefore used to optimize the reactions conditions. Indeed, $\mathrm{pH}$ conditions were adjusted, and nitrogen degassing was implemented to the preparation protocol to improve the number of ligands effectively coupled with the protein. In particular, MS analysis was performed on HSA after the thiolation reaction. Using optimal conditions, MS analysis provided a mass of $69360 \mathrm{Da}$ for thiolated HSA which corresponds to an average number of 27 thiols effectively added to the protein, and potentially available for reaction with a maleimide (Figure S3). 
We determined HSA and DTPA dn/dc to be 0.1731 and $0.1574 \mathrm{~mL} / \mathrm{g}$ respectively. The literature value of lactose is $0.150 \mathrm{~mL} / \mathrm{g} .{ }^{19}$ Exploiting both UV and RI quantification, we could quantify proteins, ligands (modifiers) and total bioconjugate MW in a straightforward manner - see Figure S4.

We achieved titration of DTPA on LACTAL and HSA-DTPA products with Eu(III) itself with the Eu / DTPA complexation pK being around 22.5 and having quick kinetics. ${ }^{18}$ When not complexed and free in solution, $\mathrm{Eu}^{3+}$ ions are quenched by $\mathrm{OH}^{-}$. Progressively increasing their quantity leads to a drastic slope break in fluorescence response when these ions stop being chelated by DTPA, as observed in Figure S5. This provides the equivalent amount of grafted DTPA, concluding the analytical steps with chelating moieties quantification. A quantity of 2 - 4 DTPA is enough for a sensitive measurement with $\mathrm{Eu}^{3+}$. Considering that $\mathrm{Eu}$ has a low quantum efficiency fluorescence and is proposed in this study as a substitute for Tc or Gd chelates to evaluate their biodistribution, it should require fewer DTPA moieties. In case of insufficient TRF signal, the $\mathrm{pH}$ and eventually the synthesis kinetics and quantities can be adjusted.

In vitro evaluation. We introduced the lactose moiety to the protein to provide a targeting ligand that would retain the protein in tissues expressing the asialoglycoprotein receptor. Since this receptor is mainly located at the apical sites of hepatocytes, ${ }^{20}$ we aimed to study the interaction between LACTAL.Eu and hepatocytes; however for this, we had to find a way of measuring this interaction.. As the protein bore a DTPA moiety for scintigraphy, we also used europium complexed DTPA to measure interaction between LACTAL.Eu and cells.

Prior to the cell experiment, it is necessary to qualitatively confirm that the targeted receptor interacts with the grafted ligand, which in this case was lactose. As shown in Figure 4A, we used the ricin agglutinin test ${ }^{21}$ and showed that the LACTAL.Eu did induce an absorbance increase (dark circle), meaning an agglutination of the ricin only when the lactose was 
present on the protein and this was reversed in the presence of free lactose in the suspension (dark square).

In vitro models are often criticized compared to vivo models for failing to replicate the precise cellular conditions of an organism. In particular, receptors expression varies greatly depending on cell culture conditions and cell lines. ${ }^{22}$ Conventional western blot and immunofluorescence provided a qualitative measure of receptor expression (Figure S6). Moreover, to acquire a fine quantification of ASGPR expression, we used flow cytometry and compared three hepatocyte cell lines (BWTG3, HEPG2 and TIB75) and 1 fibroblast cell line (3T3) - see Figure S7. The optimal percentage of ASGPR expression was obtained with HEPG2 lines was $29.4 \%$ expression. However, this parameter can be optimized by the culture conditions, particularly to amplify the internalization response demonstrated in the next step.

We loaded LACTAL.Eu and HSA-DTPA.Eu respectively onto hepatocyte (HEPG2) cells expressing the ASGPR receptor as verified. The advantage of europium in this case is that signals could be measured in the cell lysates to give quantitative data based on a preliminary calibration in the media. The specific interaction between the LACTAL.Eu and HEPG2 cell line was tested after an incubation of $4 \mathrm{~h}$ at $37^{\circ} \mathrm{C}$ as shown on Figure 4B. As expected, a significant difference was obtained with the lactose conjugated with albumin for a cells quantity of $2 \times 10^{5}$ and $4 \times 10^{5}$ per well. Finally, we assessed the toxicity of LACTAL.Eu and HSA-DTPA compound by measuring mitochondrial activity - see Figure S8. No major toxicity were observed with BNL and INH-3T3 cell lines after 24h.

In vivo evaluation. Optimizing the extraction of the biomolecule pushes the probe's limits of detection. In the case of TRF, we increased the number of parameters, including delay time, gate time and fluorescence measurement. Since TRF measurements of chelated Eu (III) are linear, the limit of detection of the spectrophotometer is directly related to noise level, background and sensitivity, i.e. slope. Though plate-readers usually have factory parameters, the literature reveals a great diversity of detection protocols when dealing with time resolved 
fluorescence. ${ }^{23}$ Figure S9 demonstrated this attempt to empirically optimize the signal. A standardized homogenization procedure optimizes the reproducibility and performances of protein extraction from tissue and avoids probe fouling due to heat. We optimized various commercial lysis buffers (Figure S10) and combined the advantages of a standard lysis buffer with a red blood cells lysis buffer, as erythrocytes absorb at $620 \mathrm{~nm}$. Papain protease was added to maximize extracted probes from proteins while avoiding eventual quenching which confirmed the relevance of a delayed fluorescence approach.

The main criterion for ex-vivo quantification is sufficient hepatic cellular uptake to confirm targeting. The use of Eu (III) time resolved assays and large Stokes shift overcame tissues' auto fluorescence, contributing to the effort of improving the limit of biodistribution quantification. We evaluated LACTAL.Eu uptake versus HSA-DTPA.Eu in a group of mice using ex-vivo TRF. As shown in Figure 5, a significant difference of $9.6 \%$ of injected dose (ID) was observed between the accumulation of the HSA-DTPA.Eu and the LACTAL.Eu probes in the liver $(\mathrm{p}<0.01, * * *)$. Other organs and blood did not display any specific response between the two products. The grafting of lactose had a significant effect on LACTAL.Eu distribution and corroborated its targeting capacities.

We finally designed a rationalized development workflow using our experience with LACTAL.Eu development combined with conjugated proteins ${ }^{16}$ and our knowledge of therapeutic protein characterization. ${ }^{3}$ This workflow highlights key criteria for the rigorous evaluation of the developed conjugate and elimination of unsatisfactory candidates. We used a conditional decision methodology (Figure 6) to optimize the procedure for in vivo characterization and ultimately facilitate clinical translation.

\section{Discussions}

Among the factors influencing biodistribution and sensitivity of the probe in the litterature, the first is the substrate type (protein, inorganic, nanoparticle...) and its chemical characteristics, such as size, shape, and charge. ${ }^{24}$ Second, the conjugation pathway used for 
ligand binding should be considered. Third, the colloidal stability and medium used for the synthesis, conservation, or in vivo injection of the biomolecular probe could impact the size distribution and number of grafted ligands with consequences for targeting function, biodistribution ${ }^{24}$ and detection. ${ }^{25}$ Finally, the specificity of the ligands and the properties of the imaging agent conjugated on the particle surface may influence the recognition and binding to the specific receptors ${ }^{26}$ increase the circulation half-life ${ }^{27}$ and define the imaging sensitivity and modalities (epifluorescence, scintigraphy, TRF or FRET...).

The signal exhibited on the mass spectra from Figure 3B demonstrated a reasonable peak width increase compared to the signal of native HSA which strongly suggests that the entity produced was not completely homogenous. Finally, the intensity of the signal was significantly lowered compared to native HSA. This observation was attributed to an increased capacity of the conjugate to absorb the energy delivered by the MALDI laser.

Though AF4-MALS-UV-RI offers a wider range of information, this technique has a nonnegligible difference with MS values, particularly for lactosylated molecules with $4 \mathrm{kDa}$ for HSA-lactose. MALS data relies on the analyte optical constants i.e. refractive index increment $\mathrm{dn} / \mathrm{dc}$ and extinction coefficient $\varepsilon$. For small mass increases, below $10 \%$ of initial mass, the optical constant of the native protein alone predominated but in our case we also needed to specify the ligand's coefficients. We compared in Table 1 (Protein Conjugate (PC) column) this alternative quantification of ligands with other ligand quantification in better agreement with MS spectrum for total mass. It seemed the modifier values were less accurate for LACTAL products which is due to the presence of two different grafting mechanisms whereas the model supposed only one. Though LS coupled to separation methods provided a low trueness value compared to mass spectrometry, the technique can be improved by knowing the modifiers' optical constants. Containing more information on conjugates homogeneity, polydispersity and sizes, this detection method constitutes a straightforward tool particularly useful for drug screening that can be used intermittently. Coupled to 
separative methods, it offers a straightforward manner to run several synthesis conditions and to obtain most of the product properties to achieve analytical validation. Depending on the nature of the probe, alternative quantifications method could be considered such as fluorescence or radioluminescence.

Lanthanide chelation allows for TRF detection, increasing measurement sensitivity and overcoming background noise issues inherent to biological tissues. This approach can motivate the development of other biomolecular agents and provide better understanding of connections between probe synthesis and biological identities. The specificity of the LACTAL.Eu for the ASGPR was demonstrated in vitro by interaction with the receptor using a ricin agglutinin test and in vitro against HEPG2 cell line. We took advantage of the TRF to perform preliminary biodistribution studies on healthy mice directly in the cell media. If we consider the previous result obtained with the LACTAL probe, ${ }^{17}$ the percent of injected dose found in the liver was close to the $29.0 \%$ found in this study. This method offers the possibility to use Eu in daily laboratory practice to pre-evaluate compounds intended for MRI, scintigraphy and biotherapies. The biodistribution results also agree with our recently published complementary approach exploiting advantages of Cy5 fluorescent dye, offering other imaging applications albeit with less sensitive detection methods and efficiency issues in biological media ${ }^{11}$. The analytical workflow implemented here was designed based on physical detections and separative techniques to determine the optimal reaction conditions and characterize the influence of the additional chemical compounds on the protein. This workflow offers a rigorous methodology for the efficient development of conjugated proteins for targeted imaging, taking advantage of absolute physical analytical characterization techniques. We also contribute to the nanomedicine community important need to understand the relation between chemical and biological identity of imaging agents ${ }^{3}$. Finally, our strategy was envisioned for LACTAL.Eu, i.e. albumin lanthanide cryptates, but could be generalized for other biomolecules with different conjugation mechanisms, targeting and imaging 
probes. ${ }^{28}$ To our knowledge, this workflow is the first published systematic protocol to optimize bioconjugates for targeted imaging.

\section{Acknowledgements}

This research did not receive any specific grant from funding agencies in the public, commercial, or not-for-profit sectors. The authors would like to thank the LIOPA optical animal imaging platform, school of Pharmacy (UMS 3612 CNRS) Life Imaging Facility of Paris Descartes University (Plateforme Imageries du Vivant), supported by France Life Imaging (grant ANR-11-INBS-0006), Infrastructures Biologie-Santé and the GDR-AIM. The authors would also like to thank INP Purpan FFF European Platform and Stéphanie Terme from Wyatt Technology France for fruitful discussions on FFF separation. For their support and fruitful discussions on the manuscript, the authors gratefully acknowledge Ruth Yoo and Nicholas DiStasio. Finally, the authors would like to acknowledge the Mass Spectrometry facility from Institut de Biologie Paris Seine for the constructive discussions on MS analysis.

Appendix A. Supplementary data

The following are the supplementary data related to this article:

\section{References}

1. Dimitrov DS. Therapeutic Proteins. Voynov, V. Totowa, NJ: Humana Press Inc.; 2012. doi:10.1007/978-1-61779-921-1_1.

2. Beck A, Goetsch L, Dumontet C, Corvaïa N. Strategies and challenges for the next generation of antibody-drug conjugates. Nat Rev Drug Discov. 2017;16(5):315-337. doi:10.1038/nrd.2016.268.

3. Coty JB, Vauthier C. Characterization of nanomedicines: A reflection on a field under construction needed for clinical translation success. J Control Release. 2018;275(February):254-268. doi:10.1016/j.jconrel.2018.02.013.

4. National Cancer Institute, Division of cancer treatment and Diagnosis; Nanotechnology Characterization Laboratory: Assay cascade protocols. https://ncl.cancer.gov/resources/assay-cascade-protocols. Accessed September 4, 2018.

5. EUNCL Nanomedicine Characterisation Laboratory : Assay Cascade. http://www.euncl.eu/about-us/assay-cascade/. Accessed September 4, 2018.

6. Roberts CJ. Therapeutic Protein Aggregation: Mechanisms, Design, and Control. Trends Biotechnol. 2014;32(7):372-380. doi:10.1111/j.1743- 
6109.2008.01122.x.Endothelial.

7. Fekete S, Guillarme D, Sandra P, Sandra K. Chromatographic, Electrophoretic, and Mass Spectrometric Methods for the Analytical Characterization of Protein Biopharmaceuticals. Anal Chem. 2016;88(1):480-507. doi:10.1021/acs.analchem.5b04561.

8. Gahoual R, Beck A, Leize-Wagner E, François YN. Cutting-edge capillary electrophoresis characterization of monoclonal antibodies and related products. $J$ Chromatogr B Anal Technol Biomed Life Sci. 2016;1032:61-78. doi:10.1016/j.jchromb.2016.05.028.

9. Berkowitz SA, Engen JR, Mazzeo JR, Jones GB. Analytical tools for characterizing biopharmaceuticals and the implications for biosimilars. Nat Rev Drug Discov. 2012;11(7):527-540. doi:10.1038/nrd3746.

10. Richard C, Chaumet-Riffaud P, Belland A, et al. Amphiphilic perfluoroalkyl carbohydrates as new tools for liver imaging. Int J Pharm. 2009;379(2):301-308. doi:10.1016/j.ijpharm.2009.05.045.

11. Lemdani K, Salmon H, Gahoual R, et al. Assessment of the targeting specificity of fluorescent albumin conceived as a preclinical agent of the liver function. Nanoscale. 2018. doi:10.1039/C8NR04163F.

12. Binnemans K. Interpretation of europium(III) spectra. Coord Chem Rev. 2015;295:1-45. doi:10.1016/j.ccr.2015.02.015.

13. Clavien P-A, Petrowsky H, DeOliveira ML, Graf R. Strategies for Safer Liver Surgery and Partial Liver Transplantation. N Engl J Med. 2007;356(15):1545-1559. doi:10.1056/NEJMra065156.

14. Marshall JS, Green AM, Pensky J, Williams S, Zinn A, Carlson DM. Measurement of circulating desialylated glycoproteins and correlation with hepatocellular damage. $J$ Clin Invest. 1974;54(3):555-562. doi:10.1172/JCI107792.

15. de Graaf W, Vetelainen RL, de Bruin K, van Vliet AK, van Gulik TM, Bennink RJ. 99mTc-GSA Scintigraphy with SPECT for Assessment of Hepatic Function and Functional Volume During Liver Regeneration in a Rat Model of Partial Hepatectomy. J Nucl Med. 2008;49(1):122-128. doi:10.2967/jnumed.107.044255.

16. Scherman D, Bessodes M, Mignet N, Chaumet-Riffaud P. Nouveaux conjugues, utilisables a des fins therapeutiques, et/ou a titre d'agent de diagnostic et/ou d'imagerie et leur procede de preparation. 2008.

17. Chaumet-Riffaud P, Martinez-Duncker I, Marty A-L, et al. Synthesis and application of lactosylated, 99mTc chelating albumin for measurement of liver function. Bioconjug Chem. 2010;21:589-596. doi:10.1021/bc900275f.

18. Byegård J, Skarnemark G, Skålberg M. The stability of some metal EDTA, DTPA and DOTA complexes: Application as tracers in groundwater studies. J Radioanal Nucl Chem. 1999;241(2):281-290. doi:10.1007/BF02347463.

19. Theisen A, Johann C, Deacon MP, Harding SE. Refractive Increment Data-Book: For Polymer and Biomolecular Scientists. Broché. Nottingham University Press; 1999.

20. Mizuno M, Kloppel TM, Nakane PK, Brown WR, Vierling JM. Cellular Distribution of the Asialoglycoprotein Receptor in Rat Liver Implications for Hepatic Accumulation of Desialylated Lymphocytes. Gastroenterology. 1984;86:142-149.

21. Zentz C, Frénoy JP, Bourrillon R. Binding of galactose and lactose to ricin. Equilibrium studies. BBA - Protein Struct. 1978;536(1):18-26. doi:10.1016/00052795(78)90047-8.

22. Nakaya R, Kohgo Y, Nakajima M, Kato J, Niitsu Y. Regulation of asialoglycoprotein receptor synthesis by inflammation-related cytokines in HepG2 cells. J Gastroenterol. 1994;29(1):24-30.

23. Mignot A, Truillet C, Lux F, et al. A top-down synthesis route to ultrasmall 
multifunctional Gd-based silica nanoparticles for theranostic applications. Chem - $A$ Eur J. 2013;19(19):6122-6136. doi:10.1002/chem.201203003.

24. Blanco E, Shen H, Ferrari M. Principles of nanoparticle design for overcoming biological barriers to drug delivery. Nat Biotechnol. 2015;33(9):941-951. doi:10.1038/nbt.3330.

25. Kijanka G, Prokopowicz M, Schellekens H, Brinks V. Influence of aggregation and route of injection on the biodistribution of mouse serum albumin. PLoS One. 2014;9(1):e85281. doi:10.1371/journal.pone.0085281.

26. Thomas E, Colombeau L, Gries M, et al. Ultrasmall AGulX theranostic nanoparticles for vascular-targeted interstitial photodynamic therapy of glioblastoma. Int $J$ Nanomedicine. 2017;12:7075-7088. doi:10.2147/IJN.S141559.

27. Gref R, Lück M, Quellec P, et al. "Stealth" corona-core nanoparticles surface modified by polyethylene glycol (PEG): Influences of the corona (PEG chain length and surface density) and of the core composition on phagocytic uptake and plasma protein adsorption. Colloids Surfaces B Biointerfaces. 2000;18(3-4):301-313. doi:10.1016/S0927-7765(99)00156-3.

28. Greg T. Hermanson. Bioconjugate Techniques, 3rd Edition. Academic P. (Audet J, Preap M, eds.).; 2013.

A.
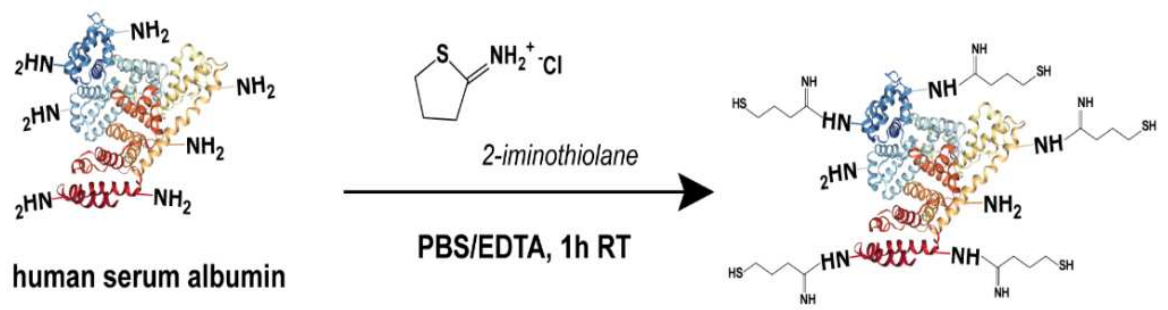

human serum albumin

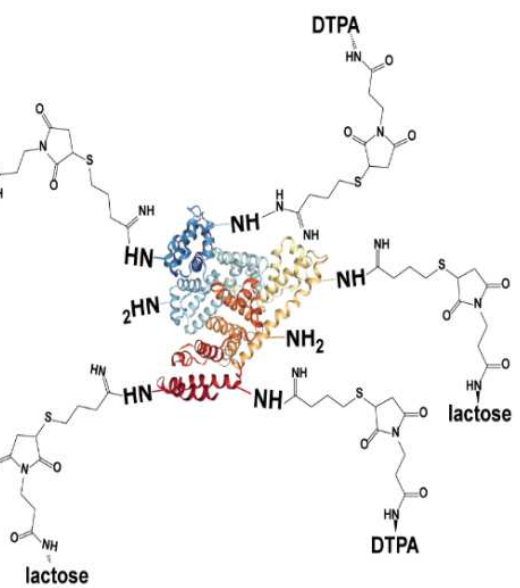

Figure 1: Schematic representation of the preparation of lactosylated DTPA serum albumin

(LACTAL). (A) Thiolating of lysine $\alpha$-amino acid groups. (B) Addition of lactose-maleimide and DTPA-maleimide are performed with a $15 \mathrm{~min}$. delay to favor ligand grafting over chelator agent. 
A

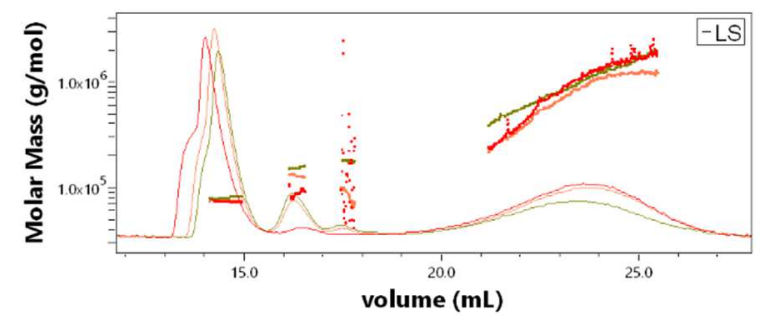

C

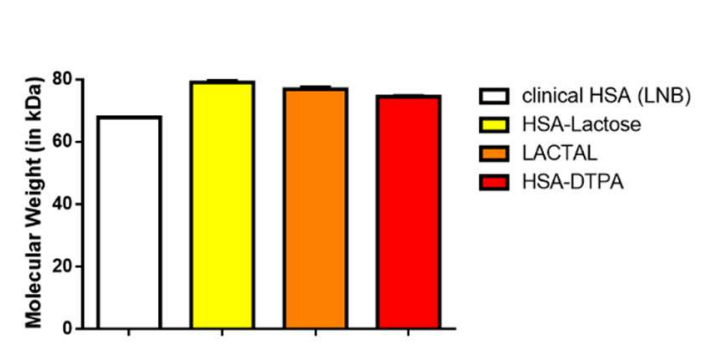

B

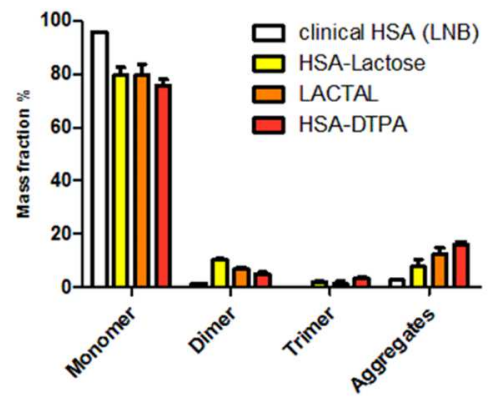

D

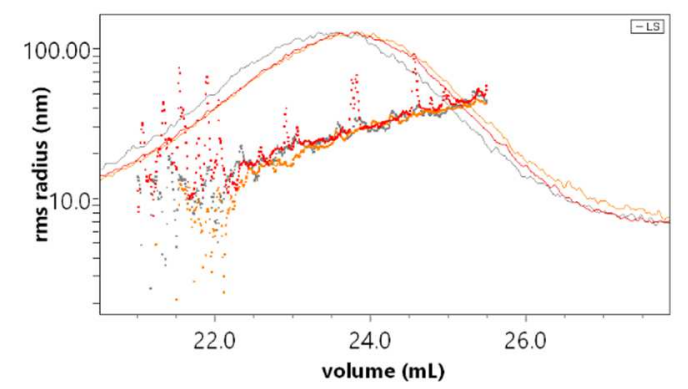

Figure 2: AF4-MALS-UV-RI analysis of HSA-DTPA, HSA-Lactose and LACTAL in red, yellow and orange respectively, $n=3$ injection per batch product. Reference HSA is represented in white in histograms. (A) Fractogram displaying molecular mass (dotted line) and light scattering (solid line) function of eluted volume (B) Oligomer and aggregates mass fractions (C) Compared products MW extracted from Zimm plot model, using albumin dn/dc (D) Compared products RMS radius (dotted line) and light scattering (solid line) function extracted function of eluted volume. 

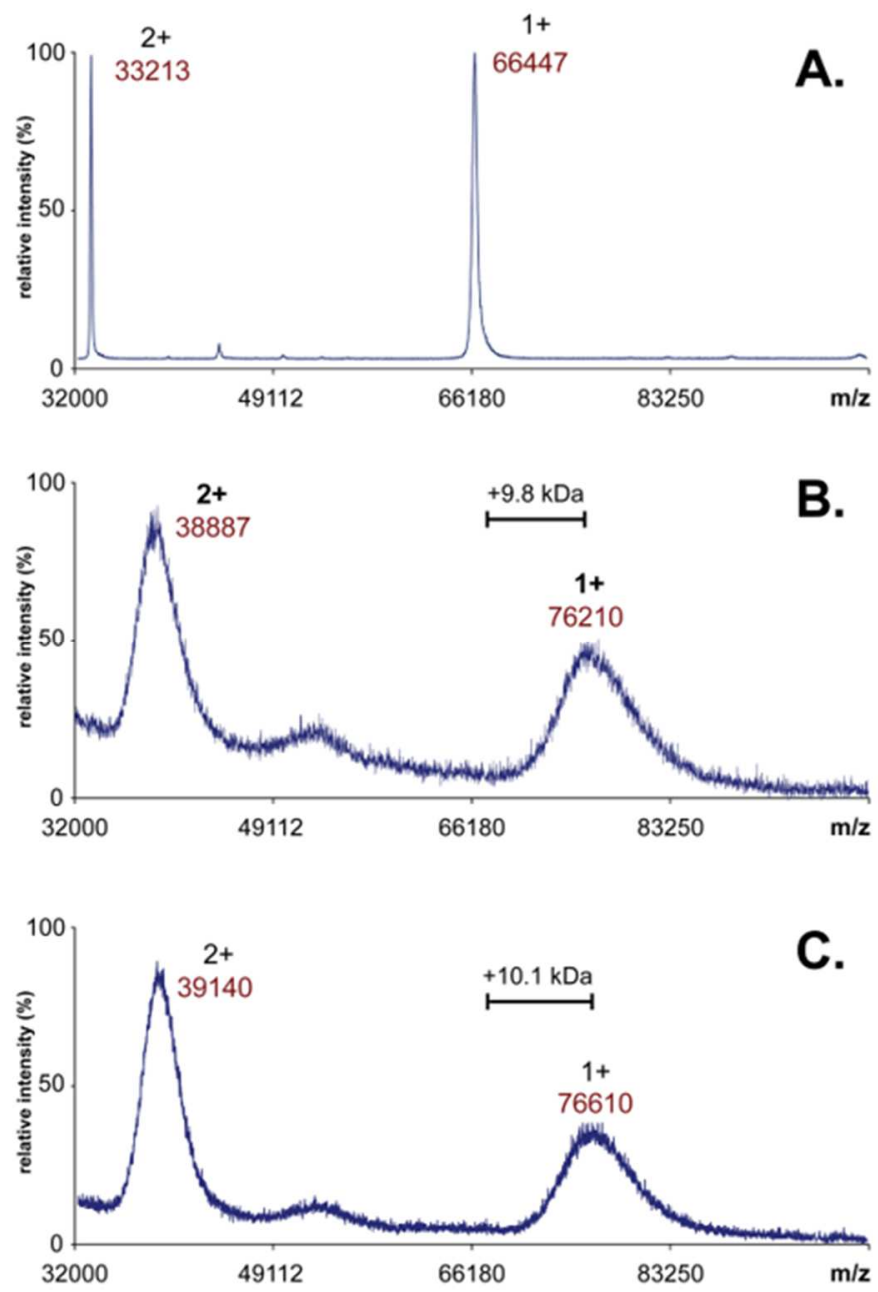

Figure 3: MALDI-TOF MS spectra corresponding to (A) clinical grade HSA, (B) HSA-

DTPA conjugate and (C) LACTAL conjugate. Average deconvoluted mass of each compound was determined considering the different charge states detected.
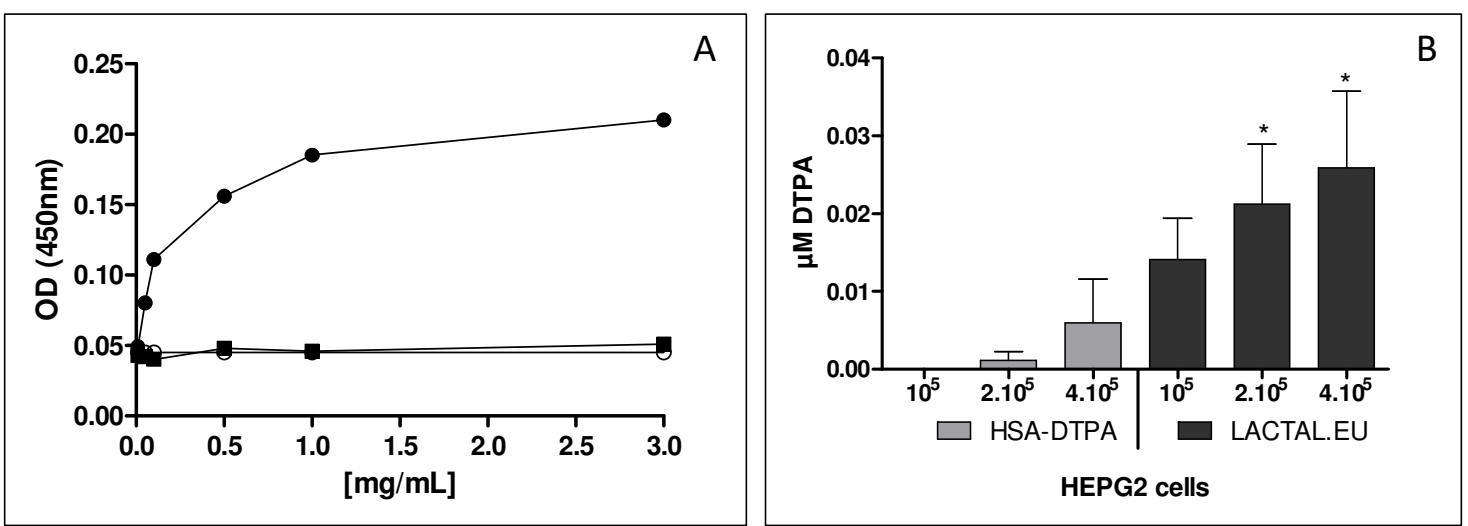
Figure 4: (A) ricin agglutinin test shows the interaction between receptor and HSA-DTPA.Eu (clear circle), LACTAL.Eu (dark circle) and LACTAL.Eu in competition with free lactose (dark square). (B) Different concentrations of HEPG2 cells were incubated with HSADTPA.Eu or LACTAL.Eu for $4 \mathrm{~h}$. at $37^{\circ} \mathrm{C}$. Europium signal was measured in the cells lysate. The amount of DTPA in the cell lysate was calculated with calibration curve realized in the same condition. Data represent the mean \pm SEM. ${ }^{*} \mathrm{p}<0.05$ (unpaired t-test, with welch's correction when the variance isn't significantly different).

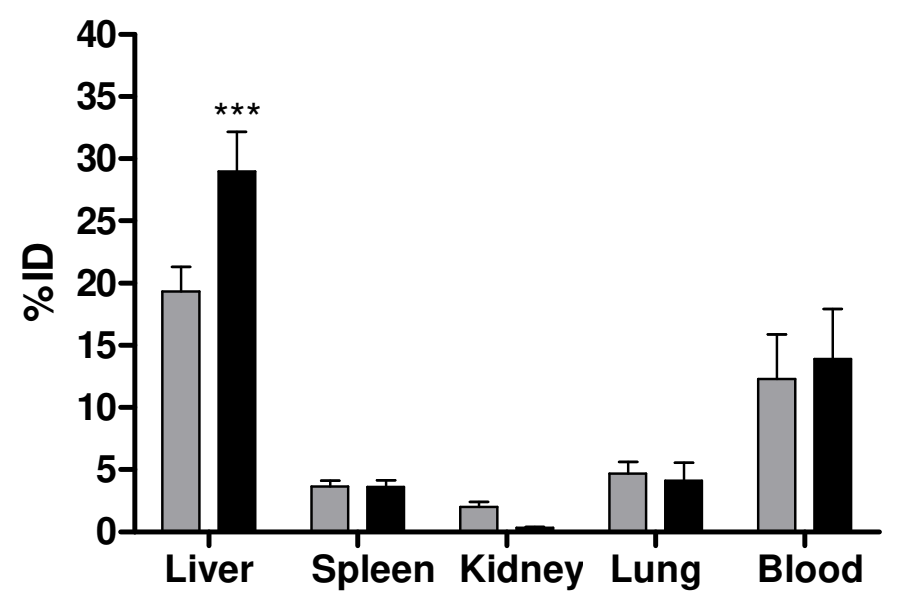

Figure 5: Biodistribution of HSA-DTPA.Eu (grey) and LACTAL.Eu (black) in Balbc/J mice twenty minutes after injection. Mean of percent of injected dose \% ID \pm SEM, two-way ANOVA with Bonferroni corrections. $n=9$ 


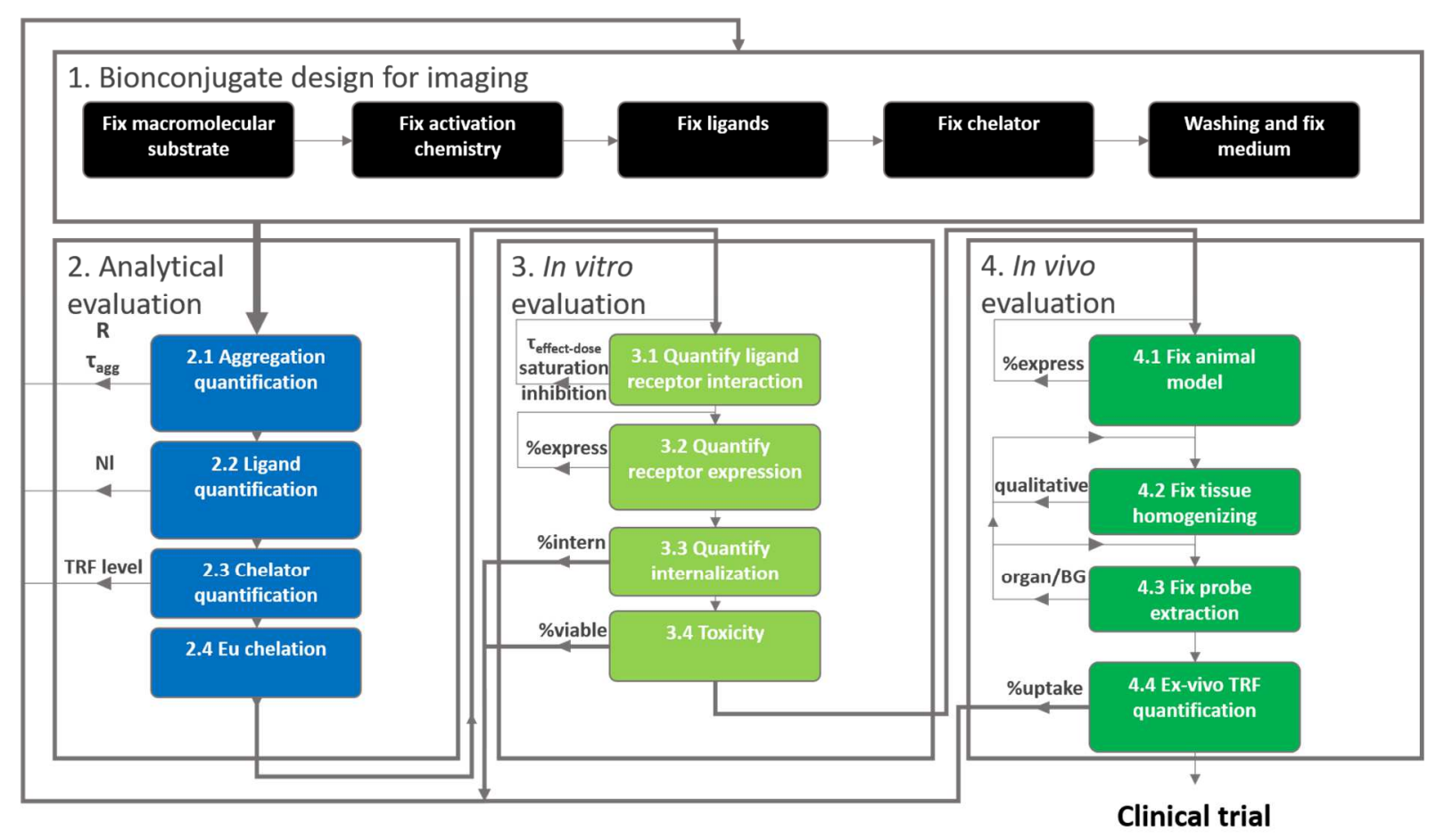

Figure 6: Schematic of implemented workflow to optimize bioconjugate development using analytical methods and europium chelates for pre-evaluation. Details regarding each step are discussed in the text.

Table 1. Molar weight increase after moieties grafting. We compare Mass Spectrometry, Light Scattering and Protein conjugate deduced from Light Scattering method.

\begin{tabular}{|c|c|c|c|c|}
\hline Product & $\mathrm{MS}[\mathrm{kDa}]$ & $\mathrm{LS}[\mathrm{kDa}]^{\mathrm{a}}$ & $\begin{array}{l}\mathrm{PC}[\mathrm{kDa}]^{\mathrm{a}} \\
\text { Total }\end{array}$ & Modifier \\
\hline HSA & 66.5 & $68.1 \pm 0.5$ & $X$ & $\mathrm{X}$ \\
\hline HSA-lactose & 75.5 & $79.5 \pm 1.2$ & $\mathrm{X}$ & $X$ \\
\hline LACTAL & 76.6 & $76.85 \pm 0.5$ & $76.7 \pm 0.5 \%$ & $13.2 \pm 2.5 \%$ \\
\hline HSA-DTPA & 75.7 & $74.5 \pm 0.56$ & $75.5 \pm 1.7 \%$ & $9+-12 \%$ \\
\hline
\end{tabular}

${ }^{\mathrm{a}} \mathrm{n}=3$ 


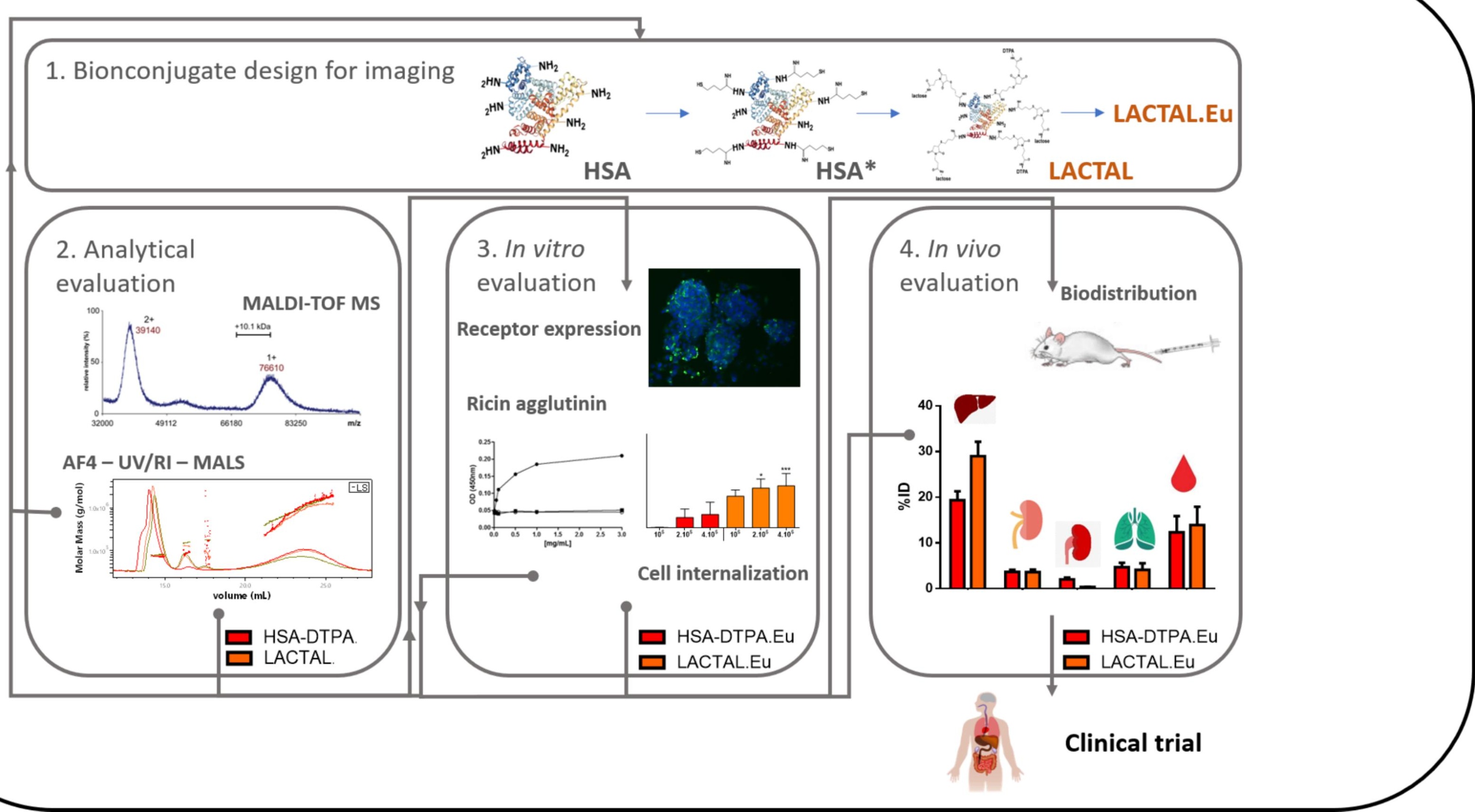

